\title{
EMPIRICAL MODELS OF THE LATITUDINAL VARIATIONS OF Te AND Ne IN THE IONOSPHERE AT SOLAR MAXIMUM
}

\author{
L.H. Brace* and R.F. Theis** \\ *Space Physics Research Laboratory, The University of Michigan, Ann Arbor, MI 48109, \\ U.SA. \\ **NASAVGoddard Space Flight Center, Greenbelt, MD 20771, U.SA.
}

\begin{abstract}
Global spectral models of electron temperature $\left(\mathrm{T}_{\mathrm{e}}\right)$ and density $\left(\mathrm{N}_{\mathrm{e}}\right)$ were produced earlier using Dynamics Explorer-2 (DE-2) Langmuir probe measurements made between 1981 and 1983. The models were derived for the fixed altitudes of 300,500 , and $850 \mathrm{~km}$. The variables in those models included spatial structure (geomagnetic latitude and longitude) and temporal variations (local time, season, UT, $F_{10.7}$, and $\mathrm{kp}$ ). Unfortunately, the DE-2 data base is not adequate to fully define all of these variables because of the eccentricity of the orbit, the slow evolution of the orbit, and the brevity of the mission (19 months). Legendre polynomials beyond order 5 could not be well enough defined to resolve small scale structures like the auroral oval, the midlatitude trough, and the equatorial anomaly, though these feature are clearly evident in the profiles from individual orbits. In the present work we take a different approach by modelling only the latitudinal variations observed in narrow altitude and local time bands and using measurements obtained during intervals short compared to a season. This approach allows the data to accurately define latitudinal terms up to 17 th order. To illustrate this improved latitudinal resolution, selected models of $T_{e}$ and $N_{e}$ at $500 \mathrm{~km}$ are compared with latitude profiles from the earlier global models and with the DE-2 measurements for the same local times and altitudes.
\end{abstract}

\section{INTRODUCTION}

The Dynamics Explorer-2 satellite (DE-2) carried a Langmuir probe to measure the electron temperature $\left(\mathrm{T}_{\mathrm{e}}\right)$ and density $\left(\mathrm{N}_{\mathrm{e}}\right) / 1,2 /$. The orbit was purely polar, with initial perigee and apogee altitudes of about $300 \mathrm{~km}$ and $1000 \mathrm{~km}$, respectively. Brace and Theis $/ 3 /$ fitted these data using $5 \mathrm{th}$ order Legendre polynomials to describe the $T_{e}$ and $N_{e}$ variations with geomagnetic latitude and local time, longitude, UT, season, $F_{10.7}$, and $\mathrm{k}_{\mathrm{p}}$. The modelling method was identical to that used earlier in modelling similar measurements from the Atmosphere Explorer and ISIS satellites /4/. Separate DE-2 models were derived for the three fixed altitudes of 300,500 and $850 \mathrm{~km}$. Unfortunately the data base did not permit the combination of all of these variables to be defined to high order, a limitation that was especially annoying for the smaller scale latitudinal structure which could be seen clearly in the individual pass plots. Features like the equatorial anomaly, the midlatitude trough, and the auroral oval were largely washed out in the global models.

To remedy this loss of latitudinal resolution, we have modelled the same DE-2 data base in a different way to suppress all of the variables except latitude. This approach has allowed 17 th order polynomials to be used, and the resulting models more faithfully reproduce the latitudinal structure evident in the DE-2 measurements. In this paper we demonstrate this improved resolution by comparing the global and the latitudinal models with each other and with the measurements. These comparisons also elucidate the nature of the $\mathrm{T}_{\mathrm{e}}$ and $\mathrm{N}_{\mathrm{e}}$ data base that is available from $\mathrm{DE}-2$. 


\section{THE DE-2 MEASUREMENTS}

The DE-2 spacecraft was launched in August of 1981. Its initial apogee and perigee altitudes were in the vicinity of 1000 and $300 \mathrm{~km}$, respectively, with apogee gradually decaying until reentry in February of 1983. The near polar orbit assured rapid drift of perigee latitude but no precession of the orbit plane. However, the local time of the orbit plane drifted by 1 hour every 15 days due to the annual movement of the Earth about the Sun. Therefore each pole to pole transit of perigee (in 50 days) occurred at a different local time. In this work we take advantage of the slow drift of local time to model the latitudinal variations of $\mathrm{N}_{e}$ and $\mathrm{T}_{\mathrm{e}}$ observed within each of these pole to pole transits of perigee.
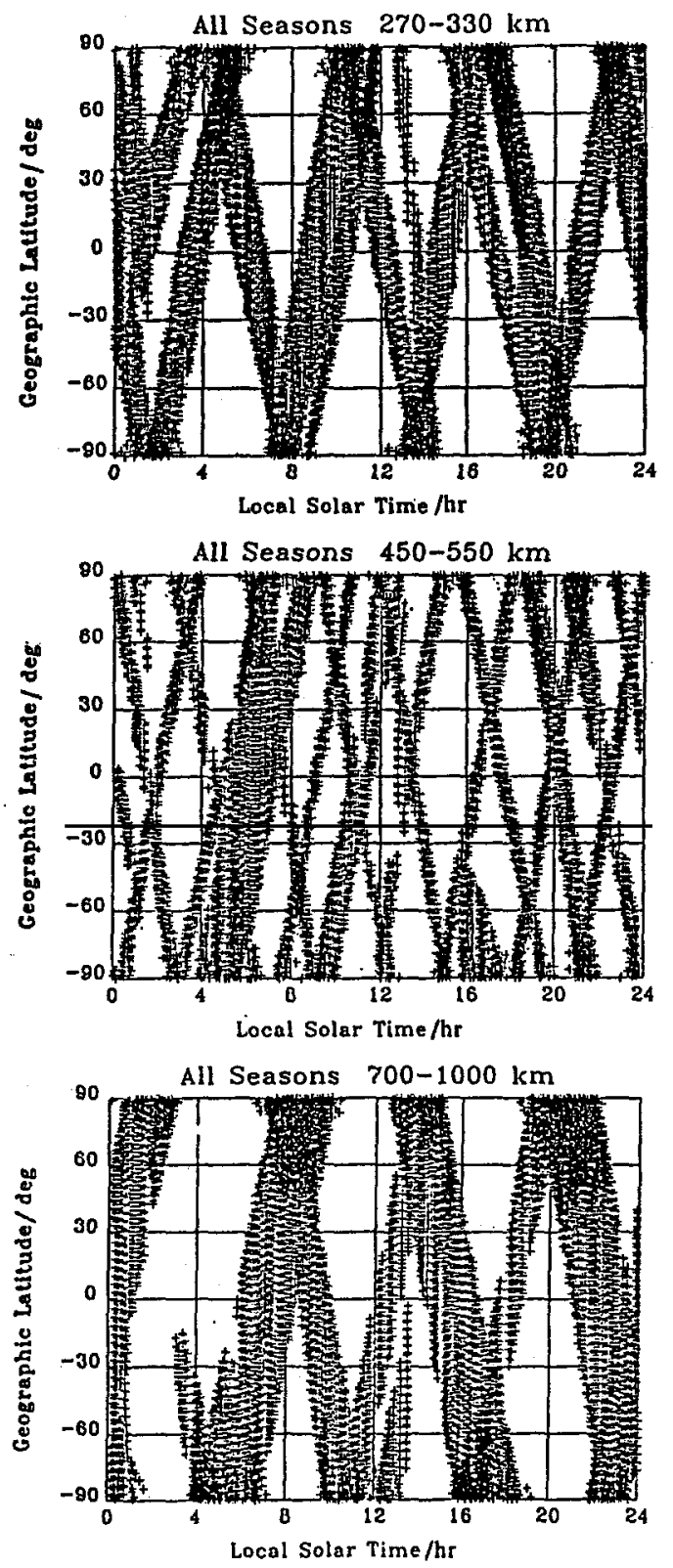

Fig 1. The motion of perigee produced this latitude and local time coverage at altitudes near 300,500 and $850 \mathrm{~km}$. Each point in these plots represents the location at which the $T_{e}$ and $\mathrm{N}_{e}$ measurements used in this work were obtained. Only the data and models from the $450-550 \mathrm{~km}$ altitude range are discussed here. 
The nature of the latitude and local time coverage is different at different altitudes. Figure 1 shows the coverage that is available in three altitude ranges; $270-330 \mathrm{~km}, 450-550 \mathrm{~km}$, and $700-1000 \mathrm{~km}$. Each plus symbol $(+)$ represents the location of the measurements that are available for modelling as described later. These altitude ranges are the same as those selected by Brace and Theis $/ /$ for their global models. Note that the local time coverage is better at middle altitudes (middle panel) than it is near perigee or apogee (upper and lower panels) because the inbound and outbound crossings through the altitude band have different local times so they tend to fill in the gaps in local time better.

\section{THE MODELLING APPROACH}

\section{The Earlier Global Spectral Models.}

As noted above, the global spectral models of Brace and Theis $3 /$ employed the DE-2 measurements made in the vicinity of 300,500 , and $850 \mathrm{~km}$. The modelling approach employed the Legendre polynomial equation (1). The data within each altitude band were first sorted into 4032 bins in latitude, longitude, and local time. The median values (one for each bin) were then used collectively to determine the coefficients $P_{1, m}$ in the $N_{e}$ and $T_{e}$ models. Actually the $\log N_{e}$ and $\log T_{e}$ were modelled.

$$
\begin{aligned}
\log _{10}\left(T_{e} \text { or } N_{e}\right)=\sum_{l=0,5}^{m=0,1} P_{1}^{m}(\operatorname{Cos} \theta)\left(e^{i m \phi}+e^{i m \gamma}\right)+ \\
\sum_{l=0,5} P_{1}^{0}(\operatorname{Cos} \theta)\left(F_{10.7}+K_{p}+e^{i S}+e^{i T}\right)
\end{aligned}
$$

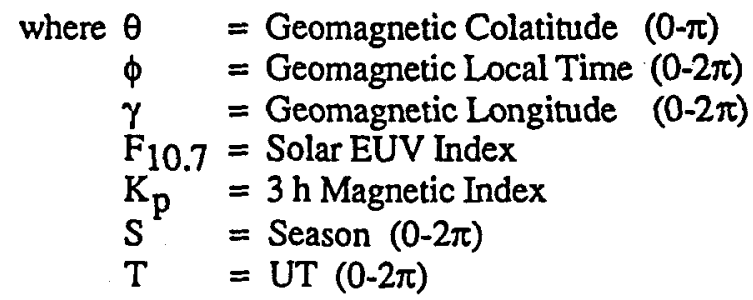

The goal of these earlier models was to define the global pattern of $\mathrm{T}_{e}$ and $\mathrm{N}_{e}$. Deviations of the data from the models were employed to determine the variations in $\mathrm{T}_{e}$ and $\mathrm{N}_{\mathrm{e}}$ with longitude, season, solar activity, and geomagnetic activity. These global models were compared with the International Reference Ionosphere model for the same conditions and reasonable agreement was found. However, the latitudinal resolution of the global models was very poor because the amount and distribution of the DE-2 data base was insufficient to accurately define the large number of coefficients included in the model.

\section{High Order Latitudinal Models}

In the present work we employed the same data base as that used by Brace and Theis $/ 3 /$ for their global models, but we use subsets of the data to better model the latitudinal $\mathrm{N}_{e}$ and $\mathrm{T}_{e}$ variations at fixed local times and altitudes. Equation (2) was used to model the data.

$$
\log _{10}\left(T_{e} \text { or } N_{e}\right)=\sum_{1=0-17} P_{1} \operatorname{Cos} \theta
$$

Equation (2) simply deletes all of the terms in equation (1) except the colatitude. As discovered by Brace and Theis $13 /$, the use of geomagnetic coordinates lead to smaller standard deviations in the coefficients. The smaller number of variables in equation (2) allowed 17 th order Legendre polynomials to be used, as compared with the 5 th order of the global models. To demonstrate the effectiveness of this approach, we modelled 21 different subsets of the data taken in the vicinity of $500 \mathrm{~km}$. The inbound or outbound measurements within each pole to pole sweep of perigee were modelled separately to reduce the local time smearing and allow more local times to be examined. 
Each model can be thought of as describing the latitudinal variations of the ionosphere at a single altitude, local time, and season. The local time is assumed to be that of the equatorial crossing of the orbit at the altitude being modelled. The data were not sorted according to $\mathrm{k}_{\mathrm{p}}, \mathrm{F}_{10.7}$ or any other parameters. The data from all longitudes were retained; i.e., we assumed that the effects of geomagnetic longitude are negligible. This assumption may be incorrect, however, as will be seen later when the models are compared with the data. In preparing the latitudinal models, we did not attempt to bin the data as was done in the global models $\beta /$ but simply used 16 second averaged values of the $T_{e}$ and $N_{e}$ measurements obtained within individual satellite passages.
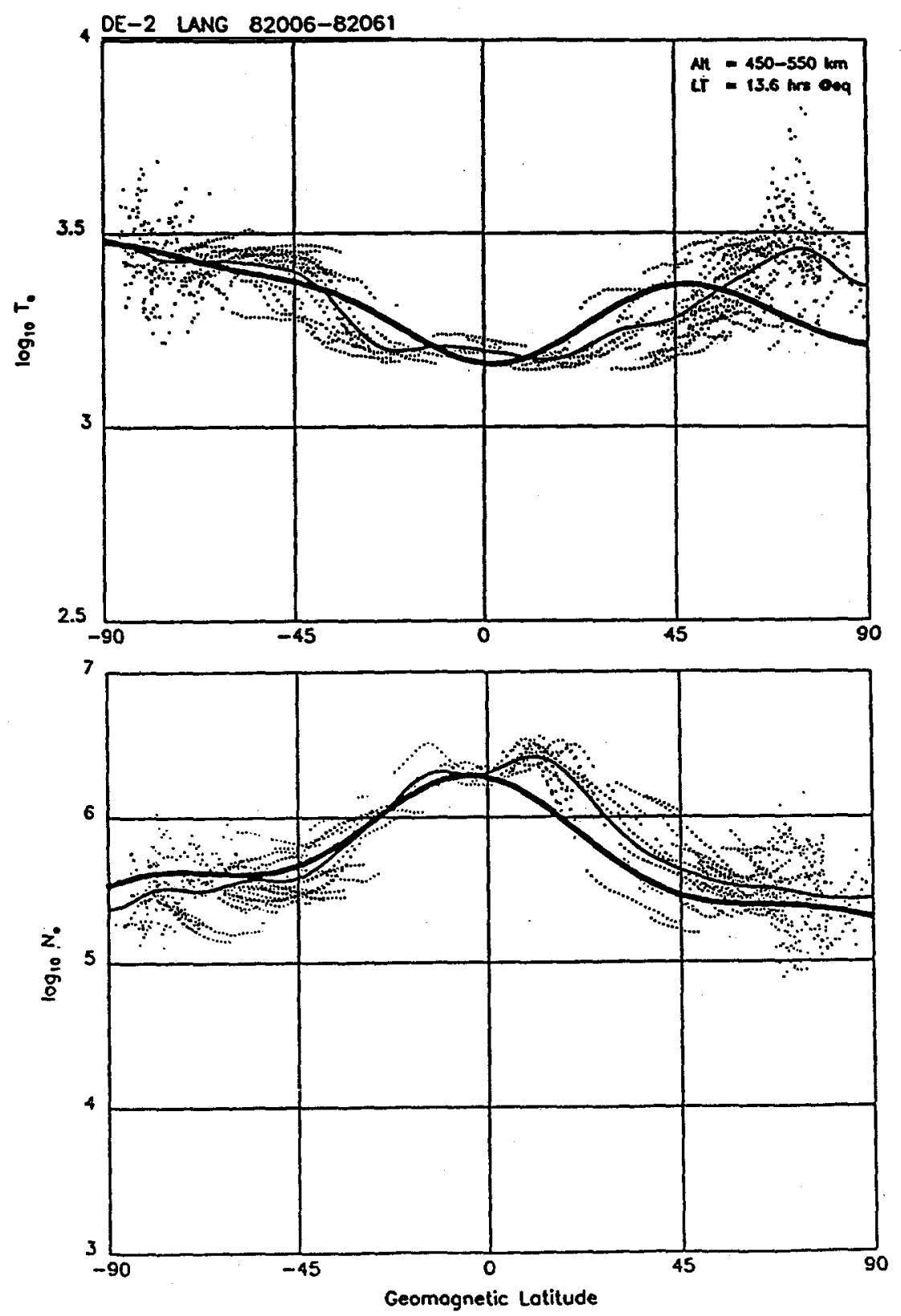

Fig 2. Latitudinal models (light line) and global models (heavy line) of $\log T_{e}$ and $\log N_{e}$ at LT $=13.6$ hours (at equatorial crossing). The points are the 16 second-averaged DE-2 Langmuir probe measurements made between days 6 and 61 of 1982 . The latitudinal models clearly track the measurements better than do the global models, although the orbit to orbit variability is large. 


\section{THE LATTTUDINAL MODELS}

As noted above, 21 subsets of measurements made between 450 and $550 \mathrm{~km}$ were modeled. Table 1 lists the coefficients of these models, but only four of them will be illustrated here. Models have not been produced for other altitude ranges. Figures 2 through 5 show representative examples of the 500 $\mathrm{km}$ models of $\log \mathrm{N}_{\mathrm{e}}$ and $\log \mathrm{T}_{\mathrm{e}}$. The corresponding global models of Brace and Theis $B /$ are shown for comparison as are the 16 second averaged DE-2 data on which the latitudinal models are based. Each model is identified by the local time of its equatorial crossing. The range of dates of the measurements (YRDAY) is labelled at the top left to help identify the season. At this time we will not comment on the geophysical significance of the features that are made evident by the higher resolution of the new models. We will simply compare the characteristics of the models and their correlation with the DE-2 measurements themselves.
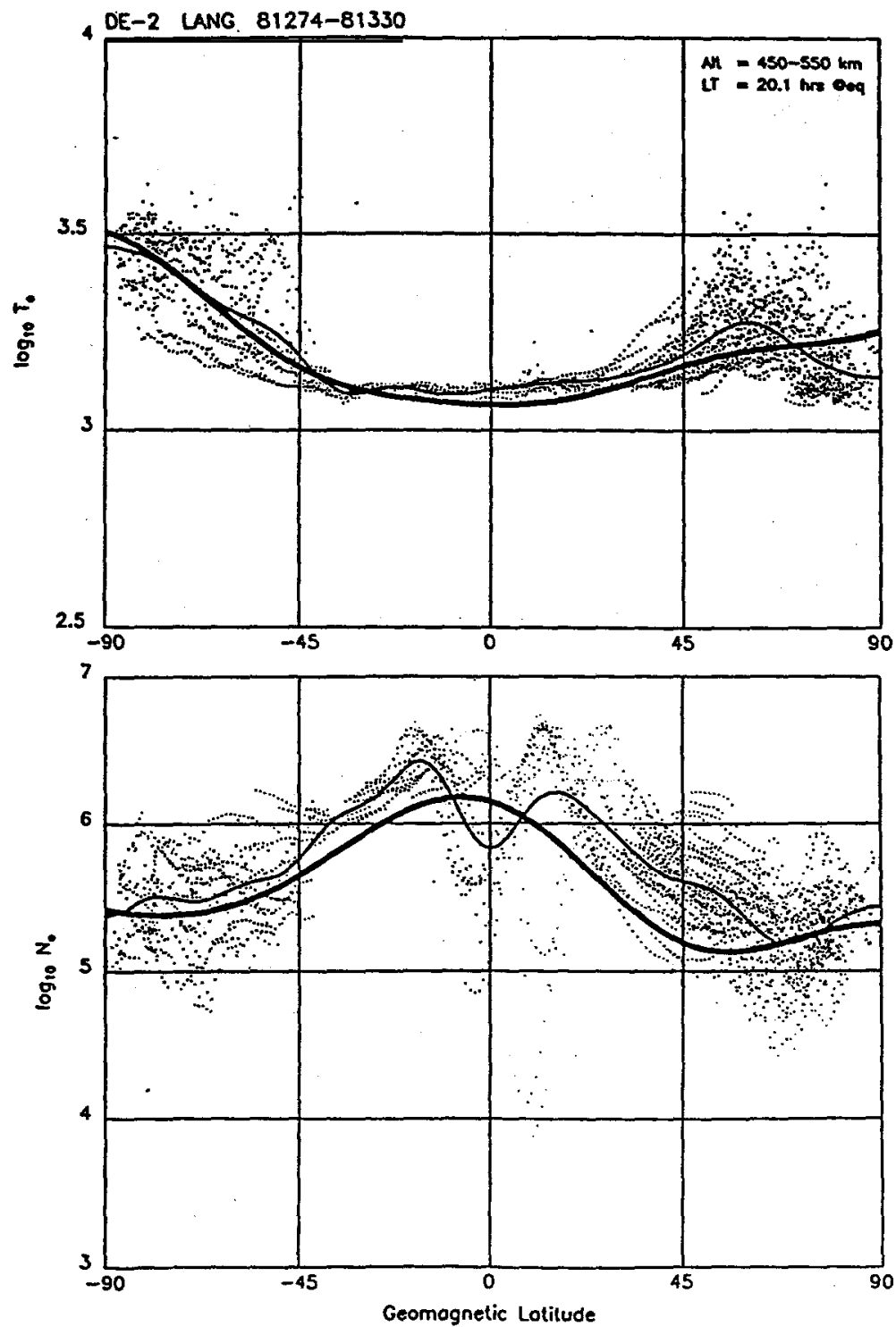

Fig 3. Same as Figure 2, but for $L T=20.1$ hours and days $274-330,1981$. The equatorial anomaly in $\mathrm{Ne}_{\mathrm{e}}$ is captured by the latitudinal model, but is washed out by the global model. The great orbit to orbit variability at higher latitudes is temporal and cannot be modelled as a latitudinal variation. 
While the global models generally agree with the latitudinal models on larger scales, the latitudinal models do a much better job of representing the smaller scale features, such as the equatorial anomaly in $\mathrm{N}_{e}$, that can be seen in Figures 2 and 3 . Very large orbit to orbit scatter is evident in the data, however. This scatter is probably associated primarily with longitudinal variations, and to a lesser extent temporal variations $\left(F_{10.7}, \mathrm{k}_{\mathrm{p}}\right.$, etc.) that are not considered in the high resolution latitudinal models. The data obtained near dawn (Figure 4) exhibits additional orbit to orbit scatter which may arise from the rapidly varying conditions in that local time sector, perhaps exacerbated by our use of geomagetic coordinates which introduce longitude-dependent solar zenith angle variations.
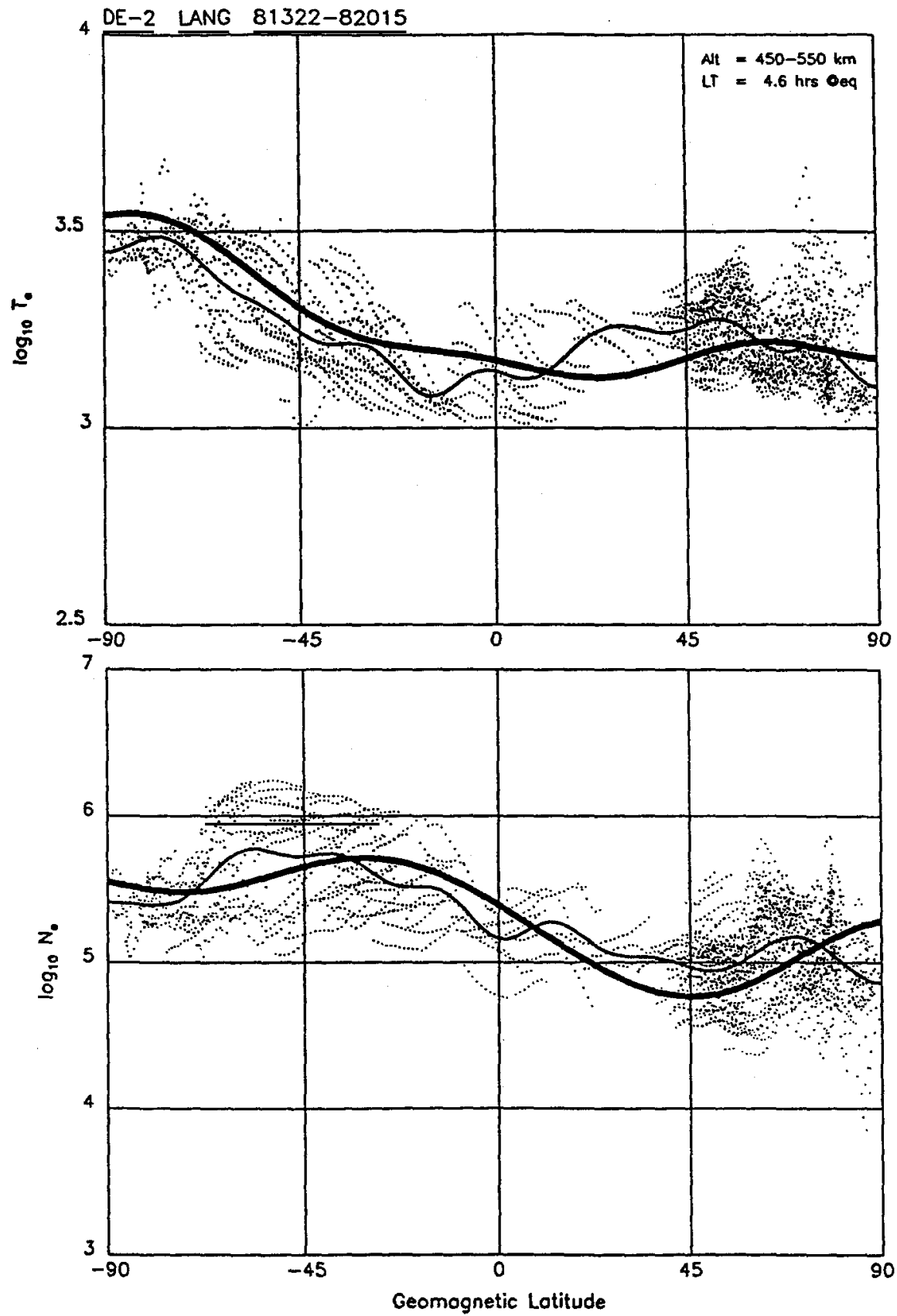

Fig 4. Same as Figure 2, but for $L T=4.6$ hours. Both $T_{e}$ and $N_{e}$ are highly variable in this time sector, but the latitudinal model captures the average structure better. 
Even the improved latitudinal resolution of the models presented here cannot capture the true shapes of latitudinal profiles of such smaller scale features as the midlatitude trough or the auroral zone. These features, though they are well resolved in the measurements from individual satellite passages, are smeared out in the latitudinal model because they move about so much from orbit to orbit. Any attempt to retain the typical shape of such features would need to find a way to remove these orbit to orbit changes, but it is not clear that such a model would be sufficiently useful to justify the effort.
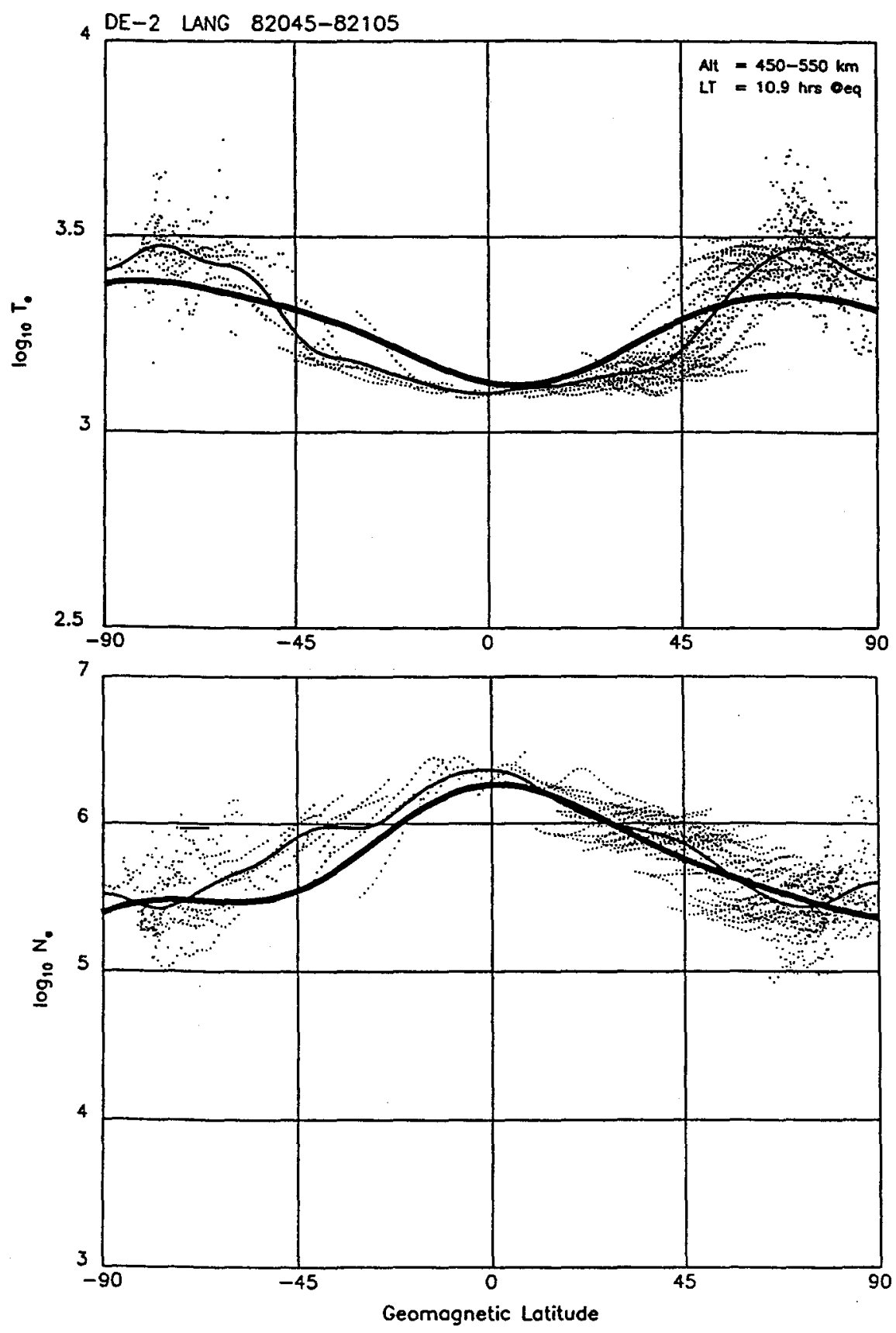

Fig 5. Same as Figure 2, but for LT=10.9 hours. $T_{e}$ and $N_{e}$ are less variable at this time and are well represented by both models. 


\section{Future Directions for Modelling the DE-2 Data.}

These simple latitudinal models could be used with the DE-2 data base to examine the remaining variables; i.e., longitude variations, $F_{10.7}$ and $k_{p}$ effects. They also could be used to generate a global mean model which contains all of the variables treated by Brace and Theis $\beta /$ in their global models, but with the advantage of higher latitudinal resolution. However, the inherently poor local time resolution of the DE-2 data base itself, evident in Figure 1, will seriously limit our ability to model the details of the diurnal variation, especially near dawn and dusk where $\mathrm{N}_{e}$ and $\mathrm{T}_{e}$ change rapidly and at both higher and lower altitudes where the local time coverage is poorer than at intermediate altitudes.

\section{SUMMARY}

The intermediate scale spatial structures that dominate the latitudinal structure of $T_{e}$ and $\mathrm{N}_{e}$ can be successfully described by Legendre polynomials by separately modelling subsets of the DE-2 data base corresponding to a narrow range of altitudes, local times, and seasons. These 17 th order models are in general agreement with the earlier 5 th order global models of Brace and Theis $3 /$, but the latter reflect only the largest scale variations. Auroral zone and mid-latitude trough structures, while evident in the measurements themselves, are not resolved by the latitudinal models because they move about in latitude. Our comparisons of the $500 \mathrm{~km}$ models with the DE-2 data on which they are based also may help future users to visualize the $\mathrm{Ne}_{e}$ and $\mathrm{T}_{e}$ data base. These data may be used in the future to examine residuals caused by other variables like longitudinal and temporal variations, specifically the response of $\mathrm{N}_{e}$ and $\mathrm{T}_{e}$ to solar EUV flux variations and geomagnetic activity.

\section{REFERENCES}

1. J. P. Krehbiel, L. H. Brace, R. F. Theis, W. R. Pinkus, and R. B. Kaplan, The Dynamics Explorer Langmuir Probe Instrument, Space Sci. Instrum. 5, 493 (1981).

2. L. H. Brace, R. F. Theis, and W. R. Hoegy, A global view of F-region electron density and temperature at solar maximum, Geophys. Res. Lett. 9, 989 (1982).

3. L. H. Brace and R. F. Theis, Global models of $\mathrm{N}_{e}$ and $\mathrm{T}_{e}$ at solar maximum based on DE-2 measurements, in press, Adv. Space Res.10, \#11, 39 (1990).

4. L. H. Brace and R. F. Theis, Global empirical models of ionospheric electron temperature in the upper F-region and plasmasphere based on in situ measurements from the AE-C, ISIS-1 and ISIS-2 satellites, J. Atmos. Terr. Phys. 43, 1317 (1981). 